CARDIOVASCULAR MEDICINE

\title{
Human fetal cardiac function during the first trimester of pregnancy
}

\author{
K Mäkikallio, P Jouppila, J Räsänen
}

Heart 2005;91:334-338. doi: 10.1136/hrt.2003.029736

See end of article for authors' affiliations

Correspondence to: Dr Juha Räsänen Department of Obstetrics and Gynaecology, University of Oulu, 90220 Oulu, Finland; juha. rasanen@oulu.fi

Accepted 27 April 2004
Objective: To investigate first trimester human fetal cardiac function in relation to cardiac volume blood flow, and peripheral arterial and venous blood flow patterns.

Methods: Transvaginal Doppler ultrasonography was performed in 16 uncomplicated pregnancies at 6+, $7+, 8+, 9+$, and $10+$ gestational weeks. The shape of the inflow waveform and the presence of atrioventricular valve regurgitation (AVVR) were noted. The outflow mean velocity (Vmean) was calculated. The proportions of the isovolumetric relaxation (IRT\%) and contraction times (ICT\%) of the cardiac cycle were defined. Ductus venosus and umbilical artery pulsatility indices (PI) were obtained.

Results: Every inflow waveform was monophasic before 9+ weeks. At 9+ weeks 11 of 16 and at 10+ weeks all waveforms were biphasic. At 7+ and 8+ weeks AVVR was documented in one case. At 9+ and $10+$ weeks AVVR was present in four and seven fetuses, respectively. Mean (SD) outflow Vmean increased between 6+ and 8+ weeks from $3.6(1.5)$ to $8.4(3.0) \mathrm{cm} / \mathrm{s}(\mathrm{p}<0.05)$. IRT\% decreased significantly from $6+$ to $7+$ weeks $(39.8(2.6)$ to $19.2(6.2), \mathrm{p}<0.001)$. ICT\% decreased between 8+ and 9+ weeks from $13.2(4.0)$ to 8.5 (2.5) ( $p<0.05)$. Ductus venosus Pls were unchanged. Umbilical artery Vmean increased between $7+$ and $10+$ weeks from $1.59(0.51)$ to $5.06(1.06) \mathrm{cm} / \mathrm{s}(\mathrm{p}<0.001)$ and Pls remained unchanged.

Conclusions: The first trimester of pregnancy is characterised by significant improvements in cardiac diastolic and systolic function with a concomitant increase in cardiac volume blood flow. At 10+ weeks AVVR is a common finding. Placental volume blood flow increases significantly with no change in the placental vascular impedance.
A lthough changes in cardiac morphology during organogenesis have been carefully investigated, knowledge of the functional development in early pregnancy is mainly based on animal experiments. ${ }^{12}$ The chick embryo model has been used to describe physiological and pathophysiological changes in the developing heart. However, these studies have often been carried out by using invasive techniques, which may disturb normal cardiovascular haemodynamic function. ${ }^{3}{ }^{4}$ Non-invasive Doppler ultrasonography has been used to describe physiological changes in the cardiac haemodynamic function of mouse embryo in both normal and abnormal conditions. ${ }^{1}$ Recent technological improvements in Doppler ultrasonography have enabled non-invasive evaluation of the physiological changes in human fetal cardiac haemodynamic function in early pregnancy. ${ }^{5}$ However, most of these studies have been performed from the late first trimester onwards. ${ }^{67}$

The aim of this longitudinal study was to characterise the development of the human fetal cardiac function in relation to fetal cardiac volume blood flow during the first trimester of pregnancy. In addition, the concomitant changes in umbilical artery and systemic venous blood flow parameters were investigated.

\section{PATIENTS AND METHODS}

Sixteen healthy women with uncomplicated pregnancies were investigated weekly in this longitudinal and prospective study between $6+$ and $10+$ weeks of gestation by transvaginal pulsed Doppler ultrasonography. Complete studies were acquired on all participants. Maternal age ranged from 2140 years (median 31 years). Mean (SD) gestational age at delivery was 40.1 (1.2) weeks and mean (SD) birth weight, Apgar scores (five minutes), and $\mathrm{pH}$ values were 3259
(390) g, 9.4 (0.7), and 7.20 (0.07), respectively. The ethics committee of the University of Oulu, Finland, approved the study protocol and all participants signed written informed consent forms.

\section{Instrumentation}

Acuson Sequoia 512 equipment (Mountain View, California, USA) was used for the examinations. The operating frequency of the transvaginal transducer (EV-8C4), which used a curved linear array format with a footprint size of $29 \mathrm{~mm}$, was $5 \mathrm{MHz}$ in pulsed Doppler mode. The cut off frequency of the high pass filter was set at minimum. The acoustic output of the system was displayed in the form of mechanical and thermal indices, which were kept at $<0.7$ and $<0.4$, respectively. The women were examined in the lithotomy position between 6.30 and 9.30 am by a single observer (KM). Gestational age was confirmed by fetal crown-rump length. ${ }^{8}$

\section{Ultrasonographic measurements}

After crown-rump length was measured, the fetal heart was identified and the sample volume $(3-4 \mathrm{~mm})$ of pulsed Doppler was placed over the entire fetal heart to obtain inflow and outflow blood velocity waveforms. The fetal heart was examined from different views to minimise the angle between the Doppler beam and the inflow and outflow regions of the heart and to obtain their maximum velocities. Maximum inflow and outflow blood velocity waveforms were recorded at a sweep speed of $100 \mathrm{~mm} / \mathrm{s}$ and their

Abbreviations: AVVR, atrioventricular valve regurgitation; ICT\%, proportion of isovolumetric contraction time; IRT\%, proportion of isovolumetric relaxation time; PI, pulsatility index; Vmean, mean velocity 
Table 1 Ultrasonographic findings in 16 fetuses

\begin{tabular}{|c|c|c|c|c|c|}
\hline & \multicolumn{5}{|c|}{ Gestational week } \\
\hline & $6+$ & $7+$ & $8+$ & $9+$ & $10+$ \\
\hline Fetal heart rate (beats $/ \mathrm{min}$ ) & $118(10)$ & $156(9)^{* *}$ & $173(8)^{* *}$ & $172(7)^{* *}$ & $167(5)^{* *}$ \\
\hline IRT\% & $39.8(2.6)$ & $19.2(6.2)^{\star *}$ & $17.1(6.1)^{\star *}$ & $14.5(1.4)^{\star *}$ & $12.5(1.1)^{* *}$ \\
\hline \multirow{2}{*}{\multicolumn{6}{|c|}{$13.4(2.3) \quad 17.3(4.7)$}} \\
\hline & & & & & \\
\hline Maximum velocity $(\mathrm{cm} / \mathrm{s})$ & $15.5(5.3)$ & $21.3(9.9)$ & $25.8(7.8)^{*}$ & $28.9(6.9)^{*}$ & $29.3(5.0)^{*}$ \\
\hline Mean velocity $(\mathrm{cm} / \mathrm{s})$ & $3.6(1.5)$ & $5.7(3.6)$ & $8.4(3.0)^{*}$ & $9.8(1.8)^{* \star}$ & $8.8(1.7)^{\star *}$ \\
\hline
\end{tabular}

time-velocity integrals were measured. The time-velocity integral, which is considered to be a measure of the length of the column of blood, was calculated by planimetry of the area underneath the Doppler spectrum. The shape of the inflow waveform was documented. When the waveform was biphasic the E (early ventricular filling) and A (filling during atrial contraction) wave peak velocities and time-velocity integrals were obtained, and their $\mathrm{A}$ to $\mathrm{E}$ ratios were calculated. The presence of atrioventricular valve regurgitation (AVVR) was documented as none, non-holosystolic $\left(\geqslant 72 \mathrm{~ms}\right.$ ), or holosystolic. ${ }^{9}$ The outflow maximum velocity was measured. The outflow mean velocity (Vmean), which is directly in proportion to volume blood flow, was calculated as follows: Vmean $=$ fetal heart rate $\times$ time-velocity integral. The proportions of the isovolumetric relaxation (IRT\%) and contraction times (ICT\%) of the total cardiac cycle were calculated..$^{10}$ For every measurement, three consecutive cardiac cycles were analysed and their mean values were used for further analysis.

Doppler shift spectra of the umbilical artery were assessed near its placental insertion. Vmean and pulsatility index $(\mathrm{PI}=$ [peak systolic velocity - end diastolic velocity]/time averaged maximum velocity over the cardiac cycle) of the umbilical artery were calculated. Blood velocity waveforms of the ductus venosus were obtained and PIs for veins $(\mathrm{PI}=$ [peak systolic velocity - velocity during atrial contraction]/time averaged maximum velocity over the cardiac cycle) were calculated. The presence of atrial pulsations in the umbilical vein was noted. The entire examination took 10 minutes at most. All data were videotaped and analysed afterwards off line by the ultrasound equipment's own cardiac measurement package. The intraobserver variability of the time interval, time-velocity integral, and absolute velocity measurements was analysed from 10 fetuses.
Table 2 Detection rates of monophasic and biphasic inflow waveforms and atrioventricular valve regurgitation (AVVR) during the study period

\begin{tabular}{|c|c|c|c|c|c|}
\hline & \multicolumn{5}{|c|}{ Gestational week } \\
\hline & $6+$ & $7+$ & $8+$ & $9+$ & $10+$ \\
\hline \multicolumn{6}{|l|}{ Inflow waveform } \\
\hline Monophasic & 16 & 16 & 16 & 5 & 0 \\
\hline Biphasic & 0 & 0 & 0 & $11^{* *}$ & $16^{* *}$ \\
\hline \multicolumn{6}{|l|}{ AVVR } \\
\hline No AVVR & 16 & 15 & 15 & 12 & 9 \\
\hline Non-holosystolic & 0 & 1 & 1 & $4^{*}$ & $6^{*}$ \\
\hline Holosystolic & 0 & 0 & 0 & 0 & 1 \\
\hline
\end{tabular}

\section{Statistical analysis}

Longitudinal changes of the measured parameters were tested by analysis of variance for repeated measurements. If significance was reached, the data were further analysed with the Fisher PLSD test. Departure from a normal distribution was assessed by means of normal plots and the Kolmogorov-Smirnov test. Groups were compared by Student's $t$ test if the data were normally distributed. Otherwise, the Mann Whitney $U$ test was selected for statistical comparison. A level of $\mathrm{p}<0.05$ was considered significant. All values are given as mean (SD).

\section{RESULTS}

The mean intraobserver variability of time interval calculations ranged from $2.7-9.5 \%$. The corresponding variability of time-velocity integral calculations varied from $6.9-7.6 \%$, and for absolute velocity measurements, from $5.0-13.2 \%$.
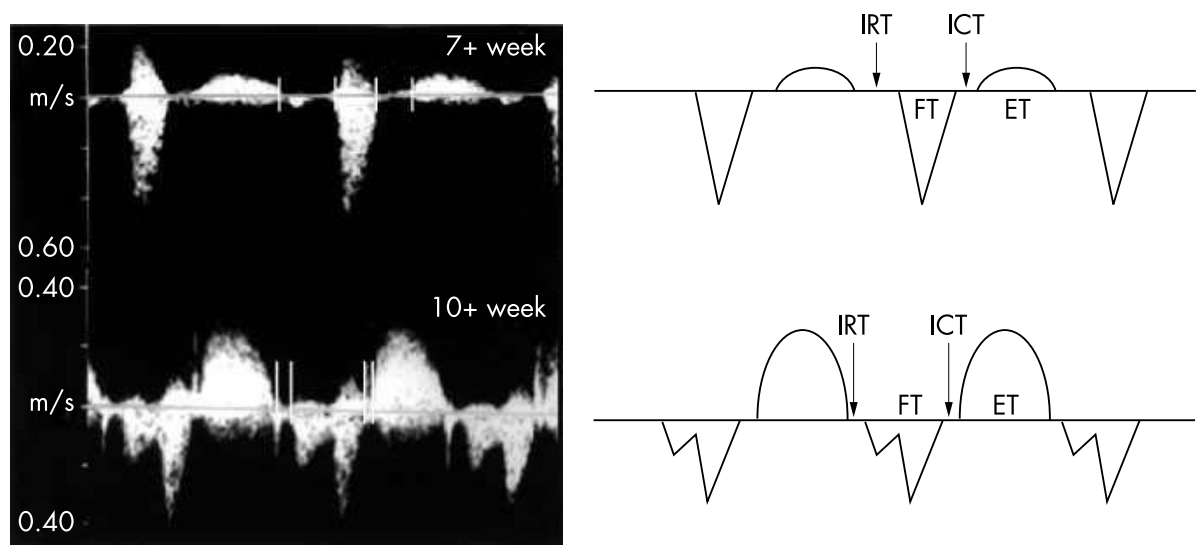

Figure 1 (Left) Ultrasonographic and (right) schematic views of isovolumetric relaxation (IRT), filling (FT),

isovolumetric contraction (ICT), and ejection (ET) times during the cardiac cycle at $7+$ and $10+$ weeks of gestation. Note the monophasic inflow at 7+ weeks and the biphasic inflow at 10+ weeks.

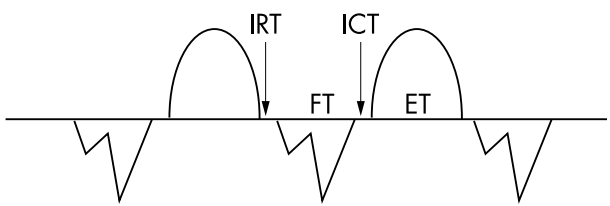



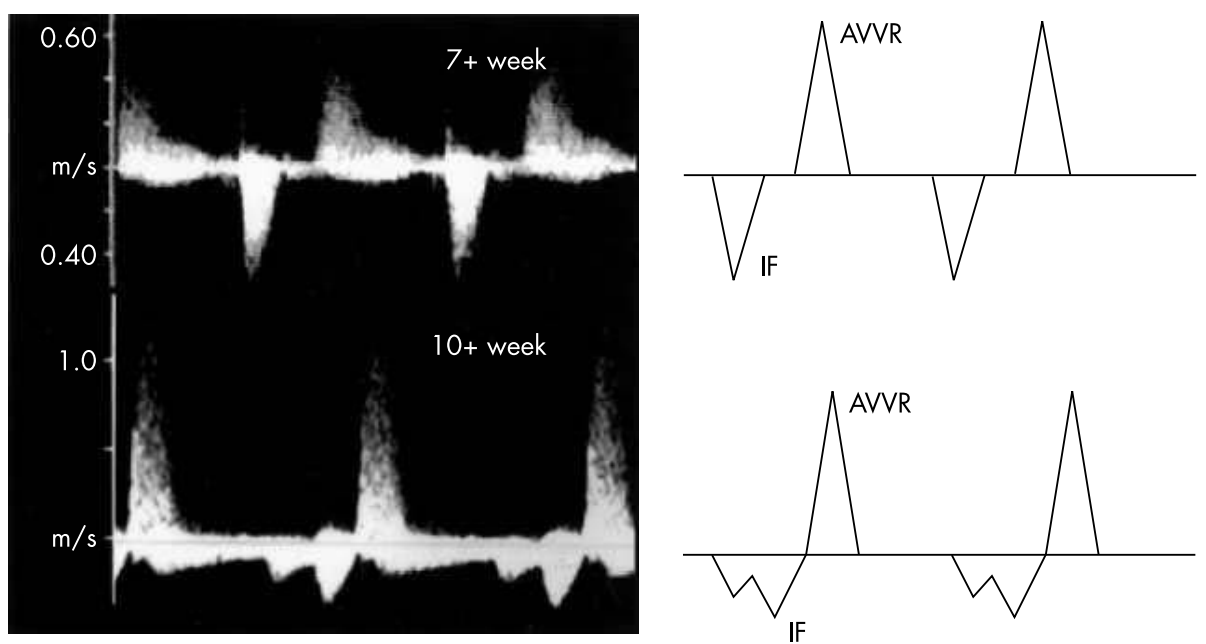

Figure 2 Non-holosystolic atrioventricular valve regurgitation (AVVR) at $7+$ and $10+$ weeks of pregnancy. Note the delay between the end of ventricular filling and the beginning of AVVR at $7+$ weeks. IF, inflow.

\section{Cardiac parameters}

Fetal heart rate increased significantly from 118 (10) beats/ min to 167 (5) beats/min between $6+$ and $10+$ weeks of gestation $(p<0.001)$ (table 1$)$. The inflow waveform was monophasic in every case until 9+ weeks of gestation. At 9+ weeks, 11 of 16 inflow patterns were biphasic and a biphasic inflow pattern was observed in every case at $10+$ weeks (fig 1 , table 2). Between 9+ and 10+ weeks, the A:E maximum velocity ratio decreased from $2.8(0.1)$ to $2.2(0.1)$ $(\mathrm{p}<0.001)$. In addition, the A:E time-velocity integral ratio decreased from $4.8(1.3)$ at $9+$ weeks to $2.9(0.7)$ at $10+$ weeks of gestation $(p<0.05)$. Table 2 gives the detection rates of AVVR at different gestational ages. At 10+ weeks, AVVR was present in $44 \%$ (seven of 16) of the fetuses. All cases of AVVR were non-holosystolic before 10+ weeks of gestation. At 10+ weeks, one of seven identified cases of AVVR was holosystolic. In fetuses with AVVR at 7+ and 8+ weeks a delay (62$66 \mathrm{~ms}$ ) was noted between the end of ventricular filling and the beginning of AVVR. At 9+ and 10+ weeks no corresponding delay was observed (fig 2).

The outflow maximum velocity and Vmean increased between $6+$ and $8+$ weeks of gestation $(p<0.05)$ (table 1$)$. IRT\% decreased from 6+ to $7+$ weeks of gestation $(\mathrm{p}<0.0001)$. At $10+$ weeks, IRT\% was less than at $7+$ weeks of gestation $(\mathrm{p}<0.05)$ (table 1$)$. ICT\% remained unchanged between $6+$ and $8+$ weeks of gestation, decreased from $8+$ to $9+$ weeks $(p<0.05)$, and did not change thereafter (table 1$)$.

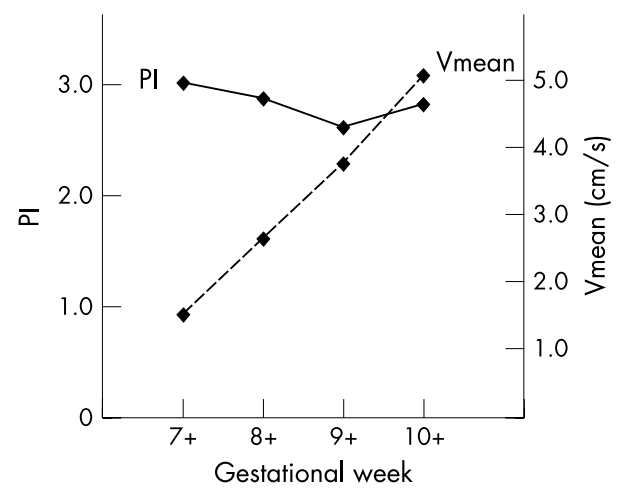

Figure 3 Changes in umbilical artery pulsatility index (PI) and mean velocity (Vmean) during the study period.

\section{Umbilical artery and ductus venosus}

Umbilical artery Vmean increased from $1.59(0.51) \mathrm{cm} / \mathrm{s}$ to $5.06(1.06) \mathrm{cm} / \mathrm{s}$ between 7+ and 10+ weeks of gestation $(\mathrm{p}<0.001)$. However, PIs remained unchanged during the same period, ranging from 3.00 (1.03) at $7+$ weeks to 2.82 (0.22) at $10+$ weeks of gestation (fig 3). During the study period, an absent diastolic blood flow pattern was documented in the umbilical artery and atrial pulsations were present in the umbilical vein in every case. The earliest documentation of ductus venosus blood flow was at $7+0$ weeks. PIs of the ductus venosus remained unchanged during the study period, varying from $1.23(0.28)$ to $1.40(0.43)$.

\section{DISCUSSION}

\section{Diastolic function of the heart}

This longitudinal study focused on the functional development of the fetal heart during the first trimester of pregnancy. According to the present results, IRT\% decreased significantly between $6+$ and $7+$ weeks of gestation with no change in ICT\%. IRT describes the time needed for the ventricle to drop its pressure from a systemic to an atrial level. During this time ventricular volume does not change. IRT\% can be used to describe diastolic function of the heart, especially during the early part of diastole. Relaxation of the myocardium is an active process dependent on the ability of myocytes to reduce the concentration of cytosolic $\mathrm{Ca}^{2+}$, which requires $\mathrm{Ca}^{2+}$ transport out of the cytosol by four pathways involving sarcoplasmic reticulum $\mathrm{Ca}^{2+}$-ATPase, sarcolemmal $\mathrm{Na}^{+} / \mathrm{Ca}^{2+}$ exchange, sarcolemmal $\mathrm{Ca}^{2+}$-ATPase, or mitochondrial $\mathrm{Ca}^{2+}$ uniport. ${ }^{11}$ Improved diastolic function of the fetal heart may be explained by morphological changes in the myocardial wall that transform it from a smooth, relatively thick shell to a porous, distensible, trabecular meshwork. ${ }^{12}$ In vitro studies of chick embryos have shown that the stress relaxation is faster in a heart with an increased amount of trabeculation in the myocardium than in the compact heart. ${ }^{13}$ At $6+$ and $7+$ weeks of gestation, the ventricular filling pattern was monophasic in every case. Therefore, the onset of ventricular filling is determined by the electrically triggered atrial contraction, and the duration of IRT may be affected by other events of the cardiac cycle before atrial contraction. However, IRT\% did not change significantly between 7+ and $8+$ weeks of gestation, when the inflow waveform was monophasic, or between $8+$ and $9+$ gestational weeks, when the inflow waveform pattern became biphasic. These results suggest that the inflow pattern itself did not significantly affect IRT\%. In addition, outflow Vmean increased between $6+$ and $8+$ weeks of gestation, suggesting a rise in the volume 
of blood flow, in particular when the diameter of the outflow tract increases at the same time. We speculate that improvement in the cardiac diastolic function in early pregnancy is important for the fetal heart to adapt to an increased volume blood flow.

\section{Systolic function of the heart}

Between 8+ and 9+ weeks of gestation, ICT\% decreased significantly with no change in IRT\%. ICT is the time interval needed for the ventricle to increase its pressure to the systemic blood pressure level, thus giving information about ventricular contractility and pressure generation during the early part of systole. ${ }^{14}$ Our results are in agreement with those of Leiva and colleagues, ${ }^{5}$ who found progressive shortening of ICT during the first trimester of pregnancy. In the late first and in the second trimester, ICT has been shown to remain unchanged. ${ }^{15}$ Before 9+ weeks of gestation, every inflow pattern was monophasic in this study, thus showing the importance of atrial contraction in maintaining adequate fetal cardiac output. In previous investigations in the late first and in the second trimester, the appearance of separate $\mathrm{E}$ and A waves in inflow has been thought to reflect changes in diastolic function of the heart-that is, a raised ventricular relaxation rate. ${ }^{14}$ In this longitudinal evaluation, IRT\% did not change during the appearance of biphasic inflow. We speculate that improved pressure generation of the ventricle enables better emptying of the ventricle, thus lowering ventricular pressure at the beginning of diastole and allowing passive filling to occur in early diastole.

\section{Atrioventricular valve regurgitation}

AVVR begins when ventricular pressure exceeds the atrial pressure and it lasts until atrial pressure again exceeds ventricular pressure. Thus, the regurgitation jet is present before ventricular ejection occurs. In addition, the maximum velocity of AVVR is higher than that of ventricular ejection because the systemic pressure is much greater than the atrial pressure. The classification of AVVR Doppler patterns has been described in human fetuses. ${ }^{9}$ Non-holosystolic AVVR was noticed in one fetus at $7+$ weeks and in one fetus at $8+$ weeks, and a delay was seen between the end of filling and the beginning of AVVR. This delay shows that the time point when ventricular pressure exceeds atrial pressure does not occur immediately after the end of the A wave. In these cases, the diagnosis of AVVR was based on much greater velocity in the regurgitation jet than in the outflow maximum velocity at $7+$ and $8+$ gestational weeks. In our study, outflow maximum velocity at $10+$ gestational weeks was similar to that reported by Wladimiroff and colleagues. ${ }^{16}$ At $9+$ and $10+$ weeks, no delay between the end of filling and the onset of AVVR was observed. This shows that at 9+ and 10+ gestational weeks ventricular pressure starts to rise immediately after the end of the A wave. This may also partially explain the decrease in ICT\% between $8+$ and $9+$ weeks of gestation.

At 9+ and 10+ weeks of gestation, AVVR was a common finding in normally developed fetuses. A dramatic increase in the volume blood flow within a relatively short time remodels the fetal cardiac three dimensional configuration, which may explain the high frequency of AVVR at 9+ and 10+ weeks of gestation. Huggon and colleagues ${ }^{17}$ found in human fetuses that apparently isolated tricuspid valve regurgitation between 10 and 14 gestational weeks was highly associated with chromosomal abnormalities. However, in $76 \%$ of cases with isolated tricuspid regurgitation, cardiac structure was not verified. In addition, the authors did not describe whether tricuspid regurgitation was holosystolic or non-holosystolic. Finally, their study population reflects a very high risk group of patients. During the second half of pregnancy, the incidence of tricuspid regurgitation is only $6-7 \%$ in normal uncomplicated pregnancies. ${ }^{9}$ In embryonic mice, AVVR was not seen in normal embryos, whereas AVVR was present in $25 \%$ of abnormal trisomy 16 embryos. In every case, AVVR was holosystolic. ${ }^{1}$ In our study, holosystolic AVVR was present in only one fetus at $10+$ weeks of gestation.

\section{Placental circulation and ductus venosus}

During the study period absent diastolic blood flow was typical in the umbilical artery as shown in earlier studies. ${ }^{18} 19$ Despite agreement with previous results showing that umbilical artery PIs remain constant during early pregnancy, our results show that dramatic changes in the umbilicoplacental circulation occur during these weeks. The finding that umbilical artery Vmean increased more than threefold, with unchanged PI, reflects an enormous increase in the umbilicoplacental volume blood flow. During this time period vascular resistance (resistance $=$ pressure/volume) seems to decrease in the placenta without affecting the umbilical artery PIs. This finding suggests that umbilical artery PIs reflect more the development and maturation of trophoblastic villous structures.

Ductus venosus blood velocity waveforms had an antegrade flow during atrial contraction already during the first trimester of pregnancy. This finding is in agreement with earlier studies that have shown that the blood velocity pattern is reversed or absent during atrial contraction in only $1-2 \%$ of normal human fetuses in early pregnancy. ${ }^{20}{ }^{21}$ Ductus venosus PIs in this study were comparable with those reported by Antolin and colleagues. ${ }^{22}$

\section{Safety aspects of ultrasonography}

Concerns about the safety of first trimester ultrasonography have been raised in recent years. ${ }^{23}$ The thermal effect is important in soft tissue-bone interfaces and it has been suggested that a temperature rise of up to $1^{\circ} \mathrm{C}$ will not have any harmful effect. It is difficult to extrapolate results obtained in vitro or in an animal model setting to in vivo conditions as a result of the difference in cooling effects. The time the embryo was exposed to pulsed Doppler ultrasonography in this study was minimised and the lowest possible output was used. To further shorten the exposure time, parameters were measured off line. The on-screen display of thermal and mechanical indices enabled the observer to continuously control the ultrasound exposure. In animal models, no haematopoietic changes have been found after frequent ultrasound exposure of embryogenic structures during organogenesis. A transient reduction in white blood cell counts in fetal blood samples has been observed after the second trimester, showing an increased thermal effect on tissue-bone interfaces by B mode as well as by triple mode ultrasonography. ${ }^{24}$ In addition, the greatest temperature increase achieved intracranially or at the muscle-bone interface was $0.6^{\circ} \mathrm{C}$ after external pulsed Doppler ultrasound exposure of 15 minutes in the second and the third trimesters. ${ }^{25}$ Recently, guidelines for research on the human fetus with Doppler ultrasonography in the first trimester have been published. ${ }^{26} 27$

In conclusion, in human fetuses during the first trimester of pregnancy, fetal cardiac functional parameters change significantly with a concomitant increase in the cardiac volume blood flow. Diastolic function improved significantly between $6+$ and $7+$ weeks of gestation as described by a shorter myocardial relaxation period during the early part of diastole. Systolic function improved greatly between $8+$ and $9+$ weeks of gestation as shown by a faster pressure generation of the myocardium during the early part of systole. During this time period a biphasic inflow pattern appeared. AVVR was a common finding at 10+ weeks of gestation. During the study period, ductus venosus and 
umbilical vein blood velocity waveform patterns did not change. In early pregnancy, the placental volume blood flow increased significantly with no change in the placental vascular impedance.

\section{ACKNOWLEDGEMENTS}

This study was financially supported by the Academy of Finland and the Finnish Gynaecological Association.

\section{Authors' affiliations}

K Mäkikallio, P Jouppila, J Räsänen, Department of Obstetrics and Gynaecology, University of Oulu, Oulu, Finland

\section{REFERENCES}

1 Gui YH, Linask KK, Khowsathit $P$, et al. Doppler echocardiography of normal and abnormal embryonic mouse heart. Pediatr Res 1996:40:633-42.

2 Wagman AJ, Hu N, Clark EB. Effect of changes in circulating blood volume on cardiac output and arterial and ventricular blood pressure in the stage 18, 24 and 29 chick embryo. Circ Res 1990;67:187-92

3 Keller BB, Hu N, Clark EB. Correlation of ventricular area, perimeter, and conotruncal diameter with ventricular mass and function in the chick embryo from stages 12 to 24 . Circ Res 1990;66:109-14.

4 Keller BB, MacLennan MJ, Tinney JP, et al. In vivo assessment of embryonic cardiovascular dimensions and function in day- 10.5 to -14.5 mouse embryos. Circ Res 1996:79:247-55.

5 Leiva MC, Tolosa JE, Binotto CN, et al. Fetal cardiac development and hemodynamics in the first trimester. Ultrasound Obstet Gynecol 1999; 14:169-74.

6 Reed KL, Meijboom EJ, Sahn DJ, et al. Cardiac Doppler flow velocities in human fetuses. Circulation 1986:73:41-6.

7 Van Splunder P, Stijnen T, Wladimiroff JW. Fetal atrioventricular flow-velocity waveforms and their relation to arterial and venous flow-velocity waveforms at 8 to 20 weeks of gestation. Circulation 1996;94:1372-8.

8 Hadlock FP, Shah YP, Kanon DJ, et al. Fetal crown-rump length: reevaluation of relation to menstrual age (5-18 weeks) with high-resolution real-time US Radiology 1992;182:501-5

9 Respondek ML, Kammermeier M, Ludomirsky A, et al. The prevalence and clinical significance of fetal tricuspid valve regurgitation with normal hear anatomy. Am J Obstet Gynecol 1994:171:1265-70.

10 Mäkikallio K, Vuolteenaho $O$, Jouppila $P$, et al. Ultrasonographic and biochemical markers of human fetal cardiac dysfunction in placental insufficiency. Circulation 2002; 105:2058-63.
11 Bers DM. Cardiac excitation-contraction coupling. Nature 2002;415:198-205.

12 Hu N, Connuck DM, Keller BB, et al. Diastolic filling characteristics in the stage 21 to 27 chick embryo ventricle. Pediatr Res 1991;29:334-7.

13 Miller CE, Wong CL. Trabeculated embryonic myocardium shows rapid stress relaxation and non-quasi-linear viscoelastic behavior. J Biomech 2000;33:615-22.

14 Weissler AM, Harris WS, Schoenfeld CD. Systolic time intervals in heart failure in man. Circulation 1968;37:149-59.

15 Van Splunder IP, Wladimiroff JW. Cardiac functional changes in the human fetus in the late first and early second trimesters. Ultrasound Obstet Gynecol 1996;7:411-5.

16 Wladimiroff JW, Huisman TWA, Stewart PA. Fetal cardiac flow velocities in the late 1st trimester of pregnancy: a transvaginal Doppler study. J Am Coll Cardiol 1991; 17:1357-9.

17 Huggon IC, Ghi T, Cook AC, et al. Fetal cardiac abnormalities identified prior to 14 weeks' gestation. Ultrasound Obstet Gynecol 2002;20:22-9.

18 Merce LT, Barco MJ, Bau S. Color Doppler sonographic assessment of placental circulation in the first trimester of normal pregnancy. J Ultrasound Med 1996;15:135-42.

19 Wladimiroff JW, Huisman TW, Stewart PA. Intracerebral, aortic, and umbilical artery flow velocity waveforms in the late-first-trimester fetus. Am J Obstet Gynecol 1992;166:46-9.

20 Murta CG, Moron AF, Avila MA, et al. Application of ductus venosus Doppler velocimetry for the detection of fetal aneuploidy in the first trimester of pregnancy. Fetal Diagn Ther 2002;17:308-14

21 Zoppi MA, Putzolu M, Ibba RM, et al. First-trimester ductus venosus velocimetry in relation to nuchal translucency thickness and fetal karyotype. Fetal Diagn Ther 2002; 17:52-7.

22 Antolin E, Comas C, Torrents $M$, et al. The role of ductus venosus blood flow assessment in screening for chromosomal abnormalities at 10-16 weeks of gestation. Ultrasound Obstet Gynecol 2001;17:295-300.

23 Duck FA. Is it safe to use diagnostic ultrasound during the first trimester? Ultrasound Obstet Gynecol 1999;13:385-8.

24 Tarantal AF, Gargosky SE, Ellis DS, et al. Hematologic and growth-related effects of frequent prenatal ultrasound exposure in the long-tailed macaque (Macaca fascicularis). Ultrasound Med Biol 1995;21:1073-81.

25 Tarantal AF, Chu F, O'Brien WD Jr, et al. Sonographic heat generation in vivo in the gravid long-tailed macaque (Macaca fascicularis). J Ultrasound Med 1993; 12:285-95.

26 Campbell S, Platt L. The publishing of papers on first-trimester Doppler. Ultrasound Obstet Gynecol 1999;14:159-60.

27 Chervenak FA, McCullough LB. Research on the fetus using Doppler ultrasound in the first trimester: guiding ethical considerations. Ultrasound Obstet Gynecol 1999;14:161.

\section{IMAGES IN CARDIOLOGY}

\section{Prussian helmet sign: a classical angiographic sign in aortic stenosis}

O

ne of the classical signs described during cardiac catheterisation in aortic stenosis is the "Prussian helmet" sign. In "pure" aortic stenosis with no concomitant aortic regurgitation, aortography often demonstrates a negative jet of radiolucent blood exiting focally from the left ventricle. In congenital aortic stenosis (secondary to bicuspid aortic valve in this particular case), there may be upward doming of the aortic valve leaflets which together with the central negative jet gives the so called Prussian helmet sign named after the lobster tail helmet used by the Prussian army. This patient underwent subsequent successful balloon aortic valvoplasty.

V Bhatia

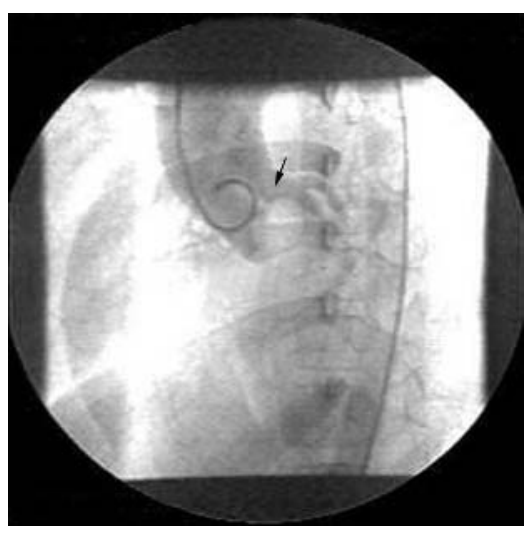

A Kumar

$S$ Tarvade

vinmonica@yahoo.co.in

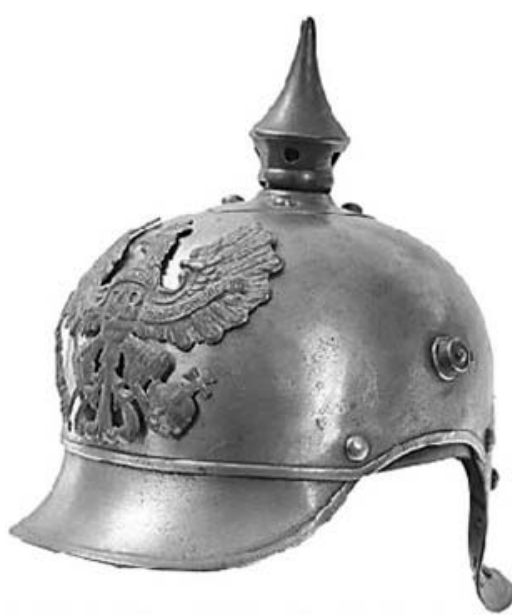

